\title{
Transparencia y su importancia para un 'accountability' y buena gobernanza en el proceso de integración regional ${ }^{1}$
}

\author{
Nicolás COBO ROMANí2
}

\begin{abstract}
"The history of the WTO is really a history of transparency. What the GATT negotiators realized in 1947 was that by creating transparency around border measures used to control trade, you could focus attention on the cost such barriers impose on our economies and negotiate them away". ROBERT MiCHael GADBAW ${ }^{3}$.
\end{abstract}

\section{INTRODUCCIÓN}

"Son injustas todas las acciones que se refieren al derecho de otros hombres cuyos principios no soportan ser publicados". E. KANT".

La transparencia y el derecho de acceso a la información pública han ganado poco a poco un reconocimiento, desde un lejano 1776, en Suecia, con la "Ley para la Libertad de Prensa y del Derecho de Acceso a las Actas Públicas". Más tarde, a través de la incorporación en las constituciones políticas en la región (Brasil, Colombia, Perú, México, Chile y Uruguay, etc.) y mediante la creación de instituciones administrativas que precisan y protegen un adecuado ejercicio del derecho, y que permiten establecer la delimitación

1 Este documento fue elaborado en el marco del Seminario SLADI 2015. Para citar el artículo: Cobo Romaní, Nicolás. (2016). Transparencia y su importancia para un 'accountability' y buena gobernanza en el proceso de integración regional, en Revista Con-Texto, n. ${ }^{\circ} 46$ EE, pp. 99-121. DOI: https://doi. org/10.18601/01236458.n46EE.06

2 Abogado de la Universidad Católica de Chile, máster en Comercio Internacional de la Universidad de Barcelona, profesor y subdirector Centro de Estudios Internacionales de la Universidad Católica de Chile(ncobo@uc.cl).

3 Transparency Disciplines in the WTO (speech given at the Services Liberalization in the Doha Round: US Industry Priorities Conference, on 12 January 2005) http://www.uscsi.org/pdf/WTO_and_Transparency 1. pdf

4 Kant, Immanuel. Sobre la paz perpetua. Madrid: Tecnos, Madrid, 1989. 
del acceso y sus causales de reservas (IFAI en México, Consejo para la Transparencia en Chile, Secretaría de Transparencia en Colombia). Recientemente, en diciembre de 2013, y siguiendo la tendencia, se aprobó una ley de transparencia en España, la que se encuentra frente a los desafíos de su implementación.

Transparencia: un DD HH. La Carta Democrática de la OEA reconoce, en su artículo $4 .{ }^{\circ 5}$, como un componente fundamental del ejercicio de la democracia, la transparencia de las actividades gubernamentales, la probidad, la responsabilidad de los gobiernos en la gestión pública, el respeto por los derechos sociales y la libertad de expresión y de prensa. La transparencia es necesaria para que el ejercicio de otros derechos humanos quede asegurado y, por ello, es considerada también un derecho fundamental y debe resguardarse como tal. Reafirmando esta concepción, la jurisprudencia de la $\mathrm{CIDH}^{6}$, en 2006, estimó que el Estado de Chile había violado compromisos internacionales al negar recursos judiciales que protegieran el derecho de acceso y la relevancia del derecho de información como parte fundamental de las garantías democráticas ${ }^{7}$.

De modo diferente, el Convenio Europeo de DD HH no lo reconoce explícitamente, y el Tribunal Europeo, al acoger algunos casos, ha optado por una norma diferente de la libertad de expresión, prefiriendo el artículo $8 .^{\circ}$, el respeto a la vida privada ${ }^{8}$. Asimismo, el Convenio 205 de 2009, sobre acceso a documentos públicos, establece una consagración ampliada del derecho y la obligación de los Estados miembros de dar la debida protección.

Asamblea General 66 ONU A/RES/66/209. El rol de Naciones Unidas en la gobernanza global se ha enfatizado en varias resoluciones ${ }^{9}$ en las que se reafirma la existencia de un verdadero "estado de derecho internacional" y de un orden internacional basado en el derecho y en el respeto de los derecho humanos, así como en la importancia de un sistema multilateral inclusivo, transparente y efectivo para enfrentar los desafíos mundiales.

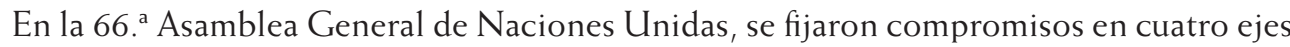
fundamentales, y a los cuales Chile adhirió, en septiembre de 2013: mayor acceso a la información y divulgación sobre las actividades gubernamentales; apoyar la participa-

5 Carta Democrática Interamericana aprobada por la Asamblea General de la OEA el 11 de septiembre de 2001, en el Vigésimo Octavo Período Extraordinario de Sesiones.

6 Reyes Claude con Estado de Chile ante la CIDH. Serie C n. ${ }^{\circ} 151$.

7 "Libertad de expresión es un elemento fundamental sobre el cual se basa la existencia de una sociedad democrática. Es indispensable para la formación de la opinión pública. Es también conditio sine qua non para que los partidos políticos, los sindicatos, las sociedades científicas y culturales, y en general, quienes deseen influir sobre la colectividad puedan desarrollarse plenamente. Es, en fin, condición para que la comunidad, a la hora de ejercer sus opciones, esté suficientemente informada. Por ende, es posible afirmar que una sociedad que no está bien informada no es plenamente libre". Cfr. Caso Ricardo Canese, supra nota 72, párr. 82, Caso Herrera Ulloa, supra nota 72, párr. 112 y Opinión Consultiva OC-5/85, supra nota 72 , párr. 70 .

8 Guichot, Emilio. Transparencia y acceso a la Información en el derecho europeo. Cuadernos Universitarios de Derecho Administrativo. Editorial Derecho Global, 2011.

$9 \mathrm{~A} / \mathrm{RES} / 61 / 39$ de 2006, A/RES//62/70 de 2008, A/RES//63/128 de 2009, A/RES//64/116 de 2010, A/RES//65/32 de 2011, A/RES//66/102 de 2012, A/RES//67/97 de 2013 y A/RES//66/256 de 2012. 
ción ciudadana, a través de la transparencia sobre la formulación de políticas y la toma de decisiones y el establecimiento y uso de vías para solicitar la opinión del público; promover estándares de integridad profesional en todos los gobiernos, y aumentar el acceso a las nuevas tecnologías, para la apertura y la rendición de cuentas.

Naciones Unidas declara que las entidades fiscalizadoras superiores solo pueden desempeñar sus tareas de forma objetiva y eficaz, si son independientes de las auditadas y se encuentran protegidas de toda influencia externa. Reconoce, asimismo, la importante función que cumplen las entidades fiscalizadoras superiores en la promoción de la eficiencia, la rendición de cuentas, la eficacia y la transparencia de la administración pública, lo que contribuye a la consecución de los objetivos y las prioridades de desarrollo nacionales, así como también de los objetivos de desarrollo convenidos internacionalmente, incluidos los Objetivos de Desarrollo del Milenio. Del mismo modo, respecto de la transparencia en áreas sensibles como compras públicas, medidas sanitarias y fitosanitarias, propiedad intelectual, compras públicas de bienes y servicios por parte de los Estados (mandato de Singapur de 1996), se han desarrollado opiniones a favor de un futuro acuerdo sobre transparencia, en concordancia con otras iniciativas, como el Grupo de Expertos en Contratación Pública del Foro de Cooperación Económica de Asia y el Pacífico (APEC).

Preeminencia del derecho en el derecho internacional y concepto de Rule of Law. Principio del ejercicio del poder estatal y otros sujetos es que se encuentre sometido a un ordenamiento jurídico aplicado de manera independiente y efectiva, en igualdad; requiere que la comunidad internacional adopte las medidas necesarias para garantizar el respeto del principio de la primacía de las normas imperativas de derecho internacional, la legalidad, la no arbitrariedad y de la transparencia ${ }^{10}$, ello en el contexto de un International Rule of Law. SIMON CHESTERMAN ${ }^{11}$ plantea para ello la presencia de cuatro elementos estructurales: i) orden internacional basado en derecho (que a su vez requiere estabilidad, previsibilidad y certeza en las relaciones), ii) aplicación uniforme del derecho internacional a todos los sujetos en un plano de igualdad, iii) prevención del ejercicio arbitrario del poder de los Estados y iv) aplicación independiente y efectiva de las normas jurídicas internacionales.

La preeminencia del derecho exige del ordenamiento jurídico internacional que se cumpla con algunos requisitos significativos, como generalidad, claridad, irretroactividad y publicidad. En ello se juega la certeza jurídica y la estabilidad de un proceso de internacionalización, muchas veces desconocido y otras, muy cuestionado. La revisión de los estándares de transparencia en el proceso de integración se vuelve, entonces, muy significativa, por su amplia presencia en convenios de integración y asociatividad, tanto de carácter regional como bilateral, y en los que la transparencia se define a través de las delimitaciones que realizan los tribunales nacionales e internacionales.

10 Villegas Delgado, CÉSAR. La preeminencia del Derecho en el Derecho Internacional. Thomson Reuters Aranzadi, 2013, p. 170.

11 Citado por Villegas Delgado. Op. cit. 
Entendemos la transparencia como el derecho a conocer lo que no se quiere mostrar por una persona o entidad que se encuentra obligada a ello. Es necesario, para un sano y democrático desarrollo de la integración comercial, que la institucionalidad de organismos multilaterales como la OMC también se abra a la información, a la inclusión y participación de los ciudadanos, que se materializa, en parte, porque los Estados se sometan a un escrutinio público, pero, además, que otras entidades, como las ONG, formen parte de los procesos de toma de decisiones (public awarness), seguimiento e información, como en el caso de la Convención de Ottawa de 1997 para la prohibición de las minas antipersonales. Un adecuado estándar de transparencia y participación no puede terminar solo en las instituciones multilaterales, sino que debe considerar cómo la información y participación desciende al nivel nacional de los ciudadanos y, en especial, cómo las autoridades locales hacen partícipes (en la medida de lo posible) y además proveen de la información (hasta donde sea necesario). Para nuestro análisis, distinguiremos tres tipos de transparencia: i) activa, a través de la información que entregan, fundamentalmente, por medio de sitios web; ii) pasiva, información entregada a solicitud de los interesados, mediante mecanismos de retroalimentación y con adecuados y simples canales para personas y entidades, sin requerir justificar un interés particular concreto, y iii) transparencia participativa o "integración líquida", que implica poder contribuir y coparticipar en los procesos de toma de decisiones en la integración; ello, en los niveles nacional, regional y multilateral.

\section{ORGANIZACIÓN MUNDIAL DEL COMERCIO (OMC)}

"Behind the terminology of the Preamble to the WTO Agreement and the many provisions agreed during the Uruguay Round, there is a living reality that affects untold millions of people. This is a crucial facet of trade that is imperfectly conveyed and understood". JULIO A. LACARTE.

\section{La transparencia como parte de la buena gobernanza}

Tradicionalmente, se considera que uno de los pilares fundamentales de la OMC es la transparencia, y ello puede parecer obvio, si observamos que el esfuerzo de los múltiples acuerdos se basa en hacer más objetivas y visibles las decisiones de los gobiernos respecto de la competencia comercial, evitando acciones fuera de los compromisos OMC. La OMC define la transparencia como el "grado de visibilidad y previsibilidad de las políticas y prácticas comerciales y de su proceso de elaboración" ${ }^{\prime 12}$, sin embargo, se trata de una concepción demasiado abierta y que no califica cómo debe ser el grado de previsibilidad. Para ROBERT WOLFE ${ }^{13}$, por su parte, el principio de transparencia es relevante para la

13 Wolfe, Robert. Regulatory Transparency, Developing Countries and the WTO. 2 World Trade Review 157 (2003), at 158 . 
OMC en dos ámbitos, interno y externo. La externa es la habilidad de la sociedad civil de seguir la labor de la OMC, mientras que la interna implica la habilidad de sus miembros, en especial los pequeños, de participar en el trabajo de la OMC. Dentro de la operatividad de la OMC se pueden señalar, como estándares de información, las reuniones de seguimiento para examinar las políticas comerciales de los países, mediante un informe a la OMC y a través de notificaciones a los demás miembros. Respecto de las prácticas no conformes con los compromisos de la OMC, como las barreras técnicas al comercio (TBT), es muy significativo mejorar los niveles de transparencia y eliminar todo trámite innecesario cuya única función sea afectar a la competencia mediante exigencias, trámites, estándares, y ellos exigen notificar los reglamentos técnicos y procedimientos de evaluación de conformidad; establecer un servicio nacional de información; el Comité de Obstáculos Técnicos al Comercio; informar a los demás miembros cualquier acuerdo (bilateral o multilateral) que incluya reglamentos técnicos, normas o procedimientos de evaluación con efectos en el comercio. También, se promueven la armonización y las equivalencias, y precisamente este es el beneficio buscado por el acuerdo entre zonas económicas como UE y EE UU, la Alianza del Pacífico, el TPP, entre otros similares, que pueden lograr importantes ventajas al armonizar o reconocer mecanismos y estándares que sean suficientes y adecuados. Los acuerdos de compras públicas (public procurement) procuran establecer normas objetivas, claras y transparentes, de modo que cualquier oferente nacional o internacional pueda participar en una posición de igualdad. Aunque la constitución de la $\mathrm{OMC}^{14}$ no fija la apertura y transparencia como un valor fundamental de la regulación comercial ni establece derechos de información para estados o individuos, hay algunos rasgos menores (Anexo 3 DSU), aunque una creciente tendencia desde $2002^{15}$ ha mejorado el acceso a información a través de las tecnologías. Desde hace tiempo, han existido críticas al proceso de toma de decisiones dentro de la OMC, calificándolo el propio PASCAL LAMY como una práctica medieval, y dentro de ello, cómo ajustar adecuadamente a políticos y oficiales, transparencia con eficacia, de manera que se entregue confianza y legitimidad ${ }^{16}$. La OMC tiene un desafío interno y externo propio, pero también lo tienen sus miembros a niveles administrativos nacionales y, en mayor medida, aquellos Estados que no cuentan con adecuados estándares democráticos que garanticen y protejan el acceso básico de los ciudadanos a este tipo de información. Hasta el año 2002, se observaba una tendencia al secretismo, que se rompe con una decisión del Consejo General ${ }^{17}$, la que dispuso que todos los documentos de la OMC debían tener publicidad. Para Gabrielle Marceau ${ }^{18}$, ello implica que todas las decisiones, reuniones, minutas, protocolos de

15 WTO, General Council. Procedures for the Circulation and De-restriction of WTO Documents, WT/L/452 (16 mayo 2002).

16 WolfE, R. Decision-Making and Transparency in the 'Medieval' WTO. Journal of International Economic Law 8(3), 631-645.

17 Decisión del 14 de mayo de 2002. (Disponible en internet: http://members.wto.org/WTO_resources/ documentation/)

18 Marceau, Gabrielle y Hurley, Mikella. Transparency and Public Participation in the WTO: A Report 
accesión, todas las leyes y regulaciones notificadas a la OMC, esquemas de los miembros y numerosos documentos de la Secretaría se deben, desde ese momento, hacer públicos en la web de la OMC. La presión ${ }^{19}$ por hacer públicas las sesiones de las disputas y permitir a los interesados hacer seguimiento en tiempo real desde las oficinas de la OMC se inició en $2005^{[20]}$. Ello, a pesar de la resistencia de terceros por abrir esta instancia a la luz. El debate del acceso a las audiencias es mucho menos contencioso que la opción del amicus curiae, ya que la observación pasiva ${ }^{21}$ causa menos recelos que la posibilidad de influir y generar consecuencias en la disputa. En varios casos se planteó el requerimiento de ONG de participar como amicus curiae, cosa que inicialmente fue considerada como no ajustada al mecanismo de Solución de Diferencias ${ }^{22}$. En 2007, la Comisión Warwick ${ }^{23}$ recomendó al Órgano de Apelación permitir a las ONG su participación como amicus curiae, que "enriquece la naturaleza y calidad de la información para los miembros del Panel en una disputa y contribuyen a la transparencia de las disputas". Entre los desafíos para la transparencia de la OMC, GABRIELLE MARCEU ${ }^{24}$ indica, precisamente, la necesidad de establecer reglas claras para la inclusión de amicus curiae, tanto de procedimiento como de fondo, lo que permite dar objetividad y previsibilidad a las partes y a los interesados en participar, como incorporar mejores mecanismos de participación y consulta para las ONG. Desde diciembre de $2006^{25}$, existe en la OMC un mecanismo provisional para la transparencia de los acuerdos comerciales regionales, en conformidad con el artículo XXIV del GATT, debiendo los Estados miembros informar a la Secretaría el inicio de toda negociación, los aspectos relativos a su alcance y la notificación a las demás partes "lo antes posible". Debido a la limitación de la regla del consenso, se le considera insuficiente e ineficaz ${ }^{26}$, ya que el encargado del monitoreo (Comité de Acuerdos Comerciales Regionales). Hay antecedentes (LEJARRAGA I. y SHEPHERD B ${ }^{27}$ ) que demostrarían que los acuerdos comerciales regionales que contengan mecanismos más profundos de transparencia aparecen como más favorables para la promoción de los flujos de comercio,

Card on WTO Transparency Mechanisms 4(1) Trade L. \& Dev. 19 (2012), p. 24.

19 Bonzon, Yves. Institutionalizing Public Participation in WTO Decision Making: Some Conceptual Hurdles and Avenues. J Int. Economic Law (2008) 11 (4): 758.

20 United States - Continued Suspension of Obligations in the EC (US - Continued Suspension).

21 Ehreing, Lothar. Public Dispute Settlement Hearings in the WTO. Journal of International Economic Law 11(4), 1021-1034, 2008.

22 US- Shrimp ab Report WT/DS58/AB/R párr. 7.8, citado por MARCEAU, GabrielLE, p. 29.

23 VAn Den BosSCHE, PETER. NGO Involvement in the WTO Journal of International Economic Law 11(4), 717-749.

24 Marceau, Gabrielle \& Hurley, Mikella. Transparency and Public Participation in the wTo: A Report Card on WTO Transparency Mechanisms 4(1) Trade L. \& Dev. 19 (2012), pp. 43-44.

25 http://www.wto.org/spanish/tratop_s/region_s/trans_mecha_s.htm

26 GaO, HenrY y Lim, C. L. Saving the WTO from the Risk of Irrelevance: The WTo Dispute Settlement Mechanism as a 'Common Good' for RTA Disputes $;$. Journal of International Economic Law 11(4), 899925.

27 LejÁrRaga, I. y SHEPHERD, B. "Quantitative Evidence on Transparency in Regional Trade Agreements". OECD Trade Policy Papers, n. ${ }^{\circ}$ 153. OECD Publishing, 2013. Disponible en internet: http://dx.doi. org/10.1787/5k450q9v2mg5-en 
alcanzando hasta un $1 \%$ de incremento por cada compromiso alcanzado en el acuerdo, además de menores costes de transacción. Los países buscan, a través de los acuerdos regionales, dar certezas a los mercados y a los inversionistas extranjeros, los que prefieren un procedimiento de toma de decisiones y en la aplicación de medidas que puedan afectar a los proveedores, y que el diseño e implementación de las reglas sea abierto y previsible.

El capítulo 11 del acuerdo NAFTA ${ }^{28}$ es considerado por GABRIELLE MARCEAU como el más trasparente, al compararlo con otros foros, y desde 2002, la transparencia en los acuerdos se ha desarrollado como política de Estado ${ }^{29}$. Todos los nuevos acuerdos consideran audiencias que deben ser abiertas al público.

LACARTE $^{30}$ nos anticipa que mayores niveles de transparencia generarán, con toda seguridad, mayores presiones y demandas a los gobiernos. Uno de los pilares de la transparencia del sistema internacional se encuentra en el artículo X del GATT 1994, que establece la obligación de publicar todas las leyes, regulaciones, decisiones judiciales y administrativas, para dar conocimiento a las partes y entidades comerciales que sean "de carácter general" ${ }^{\prime 31}$. Lamentablemente, existen varios casos de violaciones al artículo X GATT $^{32}$. También es significativo el artículo $7 .^{\circ}$ del Acuerdo MSF, que señala que, en su política sanitaria, los Estados deben notificar "las modificaciones de sus medidas sanitarias o fitosanitarias y [facilitar] información sobre sus medidas sanitarias o fitosanitarias, de conformidad con las disposiciones del Anexo B (Transparencia de las reglamentaciones sanitarias y fitosanitarias)". Puede sorprender que la jurisprudencia entre 1947 y 1980 no hizo mención ${ }^{33}$ a este texto. La aparición de un consenso relativo a los valores del good governance (transparencia, acceso a información y participación) surge más tarde $\mathrm{e}^{34}$. El Órgano de Apelación ha indicado que la obligación es extensiva a otros instrumentos que son aplicables, por lo general, y que tienen carácter similar a los instrumentos men-

"Each Party agrees to make available to the public in a timely manner all documents submitted to, or issued by, a Chapter 11 tribunal".

29 us Trade Act of 2002. Ver us Public Law 107-210 (agosto 6, 2002), 116 Stat. 996 , Div. B $f 2102$. Foreing Investment Trade Negotiating Objectives, at Part B(3), citado por MARCEAU, GabrielLE \& HuRLEY, MiKelLA. Transparency and Public Participation in the WTO: A Report Card on WTO Transparency Mechanisms 4(1) Trade L. \& Dev. 19 (2012), p. 39.

30 LACARTE, JULIO. "Transparency, public debate and participation by NGOs in the WTO: a WTO perspective"; Journal of International Economic Law 7(3), 638-686, 2004.

31 WT/DS69/AB/R 13 July 1998 (Órgano de Apelación European Communities - Measures affecting the importation of certain poultry products ).

32 Argentina - Hides and Leather, Dominican Republic - Import and Sale of Cigarettes, and us - Customs Bond Directive and EC - Selected Customs Matters. In Japan - Agricultural Products II, the Appellate Body found a violation of the transparency requirements of Article 7 of the SPS Agreement, while in Canada - Patent Term, the Panel held that the due process requirements of Article 41 of the TRIPS agreement had not been respected. 47 In Argentina - Poultry AntiDumping Duties, 48 the Panel found a violation of the public notice provisions of Article 12.1 of the Anti-Dumping Agreement ${ }_{i}$ whilst in Guatemala - Cement II. Citado por Stewart, R. y RatTon SÁnChez BADin, M. En: IILJ Working Paper 2009/7 (Global Administrative Law Series) Finalized 10/14/2009 (www .iilj.org).

33 ALA'I, PADIDEH. Transparency and the Expansion of the WTO Mandate. American University International Law Review 26, n. ${ }^{\circ} 4$ (2011): 1009-1029.

34 Informe del Órgano de Apelación sobre el asunto Japón - Productos agrícolas II, párr. 105 a 107. 
cionados explícitamente en una lista ilustrativa, y prevenir discriminaciones arbitrarias o injustificadas que impliquen restricciones al comercio. Cuando observamos los trade remedies (salvaguardias, derechos compensatorios y antidumping), se requieren mejores estándares de transparencia. En efecto, en los procesos llevados a cabo en las autoridades administrativas (con mayor o menor independencia del poder ejecutivo y, por ello, con mayor autonomía de presiones políticas), los procesos cuasijudiciales en las autoridades locales tienen escaso espacio para conocer las fundamentaciones, manejando muchas veces información sensible para la industria involucrada, que se toma en cuenta en una medida que tienen derecho a aplicar los gobiernos, con base en argumentos técnicos y en los que las posibilidades de revisión o de apelar a una entidad independiente del poder político son remotas o muy complejas.

"The significance of transparency lies not only in the original notification made by a WTO Member, but also in the interaction between Members that it stimulates ${ }^{\prime \prime 2}$. CRIS DOWNES.

JOHN H. JACKSON ${ }^{36}$, en 2001, distinguía entre los desafíos de la OMC, por una parte el de transparentar para informar al público, y otro, separado, de permitir mayor participación de las ONG y la sociedad civil, ello debido a un uso ambiguo del término "transparencia", y que se le permita a la sociedad civil la oportunidad de ser escuchada y de presentar sus perspectivas. Hoy, es claro que no basta con solo informar al público y a la institución, sino que además se debe hacer partícipes a los stakebolders en el proceso de toma de decisiones, pasando desde la transparencia activa para ganar aceptación y mejorar la legitimidad de los procesos, para lo cual es necesario identificarlos, convocarlos y escucharlos. Los desafíos de una sociedad cuya interacción y las exigencias de ser parte de la toma de decisiones ya no permiten dejar de lado y en un rol pasivo a las organizaciones sociales que han logrado encausar la representatividad de distintos grupos (etnias, sindicatos, sectores productivos pequeños y medianos, etc.).

\section{CHILE Y SU OPCIÓN POR LA TRANSPARENCIA}

En la Constitución Política de Chile, que fuera modificada en el año 2005, se fortaleció el principio de probidad con un nuevo artículo $8 .{ }^{\circ}$ : son públicos los actos y resoluciones de los órganos del Estado, así como sus fundamentos y los procedimientos que utilicen. La reserva quedó restringida a una ley de quórum calificado que podrá establecer, cuando la publicidad afecte el debido cumplimiento de las funciones de los órganos, los derechos de las personas, la seguridad de la Nación o el interés nacional. Ello implicó un reforzamiento de la ley de bases, modificada por la Ley n. ${ }^{\circ} 19.653$ de 1999 (ley de probidad) y la Ley no. 19.645, que mejoraron aspectos penales de la corrupción; artículos 3. inciso 2 y 11. bis de la Ley Orgánica Constitucional de Bases Generales de la Administración

35 Downes, C. J Int. Economic Law (2012) 15 (2): 503-524.

36 JACKSON, J.H.; The WTO 'constitution' and proposed reforms: seven 'mantras' revisited. J Int. Economic Law (2001) 4 (1): 67-78. 
del Estado, estableciéndose que la función pública debe ejercerse de modo que permita y promueva el conocimiento de los procedimientos, contenidos y fundamentos de las decisiones que se adopten en ejercicio de ella.

La doctrina identifica una "tercera generación de procedimientos administrativos" ${ }^{137}$, en la que la transparencia juega, ya no un rol de informar o de mera rendición de cuentas para el control, sino en la creación y aplicación del derecho; una cooperación públicoprivada y cooperación interadministrativa (horizontal y vertical), mediante la colaboración de la administración en la generación de normas administrativas, con una administración cooperativa o en red. Parece claro, afirma JAVIER BARNÉS VÁZQUEZ ${ }^{38}$, que una administración que no es autosuficiente, y que halla urgida el trabajo en común y en red, reclama procedimientos y actuaciones marcados por una transparencia cualitativamente distinta a la que se da en modelos tradicionales de gobierno. En la tercera generación de los procesos administrativos, se observan tanto procesos decisorios como no decisorios (de análisis e información).

Hay, asimismo, diversos compromisos internacionales asumidos por Chile en el ámbito de la probidad ${ }^{39}$, y en su cumplimiento anticipa importantes desafíos para proyectos con implicancias medioambientales o que afecten a comunidades indígenas (Convenio 169 OIT $)^{40}$, que consideran etapas de consulta y participación en decisiones de políticas que puedan afectarles, y hacen conveniente y exigible ampliar al máximo la apertura, restringiéndola a causales muy claras y bien delimitadas.

Open Government Partnership. Estamos ante una nueva etapa internacional que promueve el Gobierno Abierto $^{41}$, la que debe incluir todos los procesos de decisión en la integración regional (negociaciones, compromisos asumidos, implementación, aplicación de estándares de reservas limitados).

El acceso a la información y lo internacional. Ha sido un argumento habitual que dentro de los temas sensibles y que exigen un mayor grado de reserva están los asuntos internacionales. El Reglamento Europeo 1049/2001 consagra dentro de las excepciones (a.4) que permiten denegar el acceso a documentos que supongan un perjuicio a la pro-

37 García Macho, Ricardo (ed.). Derecho Administrativo de la información y administración transpa-

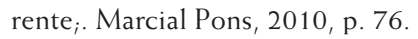

38 García MaCHO, Ricardo (ed.). Derecho Administrativo de la información y administración transparente, . Marcial Pons, 2010, p. 77.

39 Convención Interamericana Contra la Corrupción, adoptada en Caracas, Venezuela, el 29 de marzo de 1996 y promulgada por D.S. n. ${ }^{\circ} 1.879$, RR.EE. (D.O. 2 de febrero de 1998); Convención para Combatir el Cohecho a Funcionarios Públicos Extranjeros en Transacciones Comerciales Internacionales y su Anexo, de la Organización para la Cooperación y el Desarrollo Económico (OCDE), adoptada en París, Francia, el 17 de diciembre de 1997 y promulgada por D.S. n. ${ }^{\circ}$ 496, RR.EE. (D.O. 30 de enero de 2002); Convención de las Naciones Unidas contra la Delincuencia Organizada Transnacional, adoptada en Palermo, Italia, el 15 de diciembre de 2000 y promulgada por el D.S. n. ${ }^{\circ} 342$, RR.EE. (D.O. 16 de febrero de 2005); Convención de las Naciones Unidas en contra de la Corrupción, adoptada en Nueva York el 31 de octubre de 2003 y promulgada por el D.S. n. ${ }^{\circ} 375$, RR.EE. (D.O. 30 de enero de 2007).

40 Artículos 6. y 7. del Convenio de la OIT, vigente en Chile desde 2009.

41 Disponible en internet: http://www.opengovpartnership.org/ 
tección de determinadas áreas sensibles, las relaciones internacionales, la seguridad, los temas de defensa y la política financiera, monetaria y económica. Lo que es coincidente con la excepción que se consigna el art 21 no. 4 de la ley de transparencia chilena ${ }^{42}$, si, en especial, se afecta el interés nacional, ya se trate de las relaciones internacionales, los intereses económicos o comerciales del país. En Chile se debatió algo interesante respecto de conocer los honorarios de los asesores del Estado en un litigio internacional ante la Corte de La Haya, entonces pendiente de resolución. Se aceptó en la instancia del Consejo ${ }^{43}$ el acceso a antecedentes, pero luego dicha resolución fue revocada por la Corte Suprema, ya que se estimó que la información requerida "dicen relación directa con la preparación de informes, redacción de documentos, argumentaciones, en pleno desarrollo, en la etapa previa a los alegatos, de tal forma la naturaleza de los servicios requeridos-jurídicos y especializados- son de carácter confidencial", siendo prerrogativa de los Estados mantenerla en reserva. En definitiva, fue la Corte Suprema, en recurso de queja, la que confirmó que sí correspondía la reserva "ratifica[ndo] que corresponde a la autoridad política la calificación del interés nacional en materia de Relaciones Internacionales y además, reiteró el carácter secreto de la relación entre el Estado de Chile y los abogados que lo representan, ratificando la primacía del secreto profesional, también contenido en tratados internacionales". La Corte Suprema ${ }^{44}$ estimó que tratándose de una facultad exclusiva del Presidente, la conducción de las relaciones internacionales y del interés nacional, solo compete a aquél la renuncia de esta reserva.

La preocupación por trasparentar las restricciones en el comercio está presente en varias entidades.

La OCDE ha expresado orientaciones sobre la necesidad de transparentar las restricciones a las exportaciones, mediante un inventario de medidas de restricciones, lo que da una visión compresiva de impuestos, cuotas, prohibiciones y todo tipo de restricciones a las materias primas y commodities agrícolas, FLIESS B., 2014 ${ }^{45}$. Transparentar las restricciones de importaciones supone una comunicación pública efectiva, ofreciéndoles la oportunidad de participación en el proceso de toma de decisiones a los grupos de interés y un trato justo en los procedimientos de la administración. Hay, asimismo, compromisos asumidos en APEC. En noviembre de 2010, se firmó la declaración de Estrategia de Crecimiento de los Líderes de APEC, para avanzar en la modernización de los mercados mediante una Nueva Estrategia de APEC para la Reforma Estructural, a través de mercados más abiertos, transparentes y competitivos, y una regulación más efectiva de los mercados y el desarrollo de las pyme, además de otras áreas prioritarias.

42 Ley 20.285

43 Amparo Rol C-1553-11.

44 Causa Rol n. ${ }^{\circ} 13510-2013$

45 FLIESS, B. Transparency of Export Restrictions: A Checklist Promoting Good Practice. OECD Trade Policy Papers, n. ${ }^{\circ}$ 164. OECD Publishing, 2014. Disponible en internet: http://dx.doi.org/10.1787/5jz417hqk38qen 
El Portal Transparencia Chile ${ }^{46}$, además de otras iniciativas ${ }^{47}$, al que se sumaron más de 20 empresas del Estado y que no se encontraban originalmente obligadas por la ley de transparencia que fue concebida para el Ejecutivo...

La Ley 20.500, de asociaciones y de participación ciudadana, crea los Consejos de la Sociedad Civil, y una nueva institucionalidad que promueve la participación de la sociedad civil, universidades, ONG, think tanks, en materias de interés ciudadano en las que se requiere conocer la opinión de las personas. Ellas se reúnen, de acuerdo con su reglamento, algunas veces en el año, se les reportan los avances de las negociaciones y se informa respecto de temáticas que son de interés de las entidades convocadas. Cada ministerio desarrolló consultas ciudadanas y estableció la creación de una instancia de reunión con una periodicidad establecida para los Consejos de la Sociedad Civil. En particular, Direcon realizó consultas relativas al avance de las negociaciones comerciales ${ }^{48}$, las que implicaron un insumo que establece un canal de diálogo y responder a las inquietudes, así como aclarar dudas existentes. En los EE UU, se han establecido recientemente (febrero de 2014) símiles de participación ciudadana en el Public Interest Trade Advisory Committee $\left(\right.$ PITAC $^{49}$ ), que entregan asesoría al U.S. Trade Representative (USTR), incluyendo salud pública, desarrollo internacional y protección del consumidor, entre otras, y apoyar al Presidente, al USTR y al Congreso, con opiniones sobre los beneficios o desventajas para la promoción de los intereses de EE UU, mediante el Federal Advisory Comitee Act $\left(\right.$ FACA $\left.^{50}\right)$, que deberá ser integrado por la industria, la agricultura, servicios, inversiones, defensa y otros intereses que incluyan a los pequeños empresarios, para dar asesoría.

Direcon y la participación ciudadana. En una posmodernidad con una sociedad líquida (Z. BAUMAN), se hace necesario reflexionar respecto de cuáles son los desafíos que implica en otras políticas públicas, como la integración económica, en sus fases de negociación, aprobación e implementación, cómo se va a hacer partícipes a los ciudadanos. Direcon ${ }^{51}$, a través de una política de mayor participación en la gestión pública y acercamiento a los gobiernos locales con el gobierno central, quiere ofrecer este acceso y participación, por medio de cuatro mecanismos: acceso a información relevante, consultas ciudadanas, cuentas públicas participativas y consejo de la sociedad civil. Este consejo supone un mecanismo de participación consultivo, que permite un diálogo y que convoca a las entidades no gubernamentales y académicas vinculadas con las temáticas. Desde el 20 de noviembre de 2012, reúne 27 entidades de diversa índole, para el conocimiento, reporte y diálogo informativo de materias relativas a negociaciones

46 Disponible en internet: http://www.portaltransparencia.cl/PortalPdT/

47 chileatiende.cl y datos.gob.cl

48 Disponible en internet: http://www.direcon.gob.cl/2014/06/director-de-direcon-destaco-proceso-detransparencia-institucional-en-reunion-delconsejo-de-la-sociedad-civil/

49 Section 135 of the Trade Act of 1974 (19 U.S.C. 2155(c)(1)) and the Federal Advisory Committee Act (5 U.S.C. App. 1).

50 Disponible en internet: http://www.gpo.gov/fdsys/pkg/uSCODE-2010-title5/html/usCODE-2010-title5app-federalad.htm

51 Disponible en internet: http://www.direcon.gob.cl/participacion-ciudadana/ 
comerciales, implementación de los tratados de libre comercio, servicios y programas para la promoción de exportaciones y participación de Chile en organismos económicos multilaterales y cuyo funcionamiento y composición está regulado ${ }^{52}$. En el artículo $12^{53}$, se indican los temas de interés ciudadano que se deben someter a la consideración del Consejo: "negociaciones comerciales; implementación de los tratados de libre comercio; servicios y programas de promoción de exportaciones y participación del país en Organismos Económicos Multilaterales".

Negociaciones TPP. Las nuevas etapas que vive la sociedad hicieron necesario mejorar los niveles de información de transparencia activa a través de páginas web de las entidades que tienen la responsabilidad de las negociaciones, el seguimiento y el monitoreo $\left(\right.$ Direcon $\left.^{54}\right)$. Hoy, en las últimas negociaciones del Transpacific Partnership, que convoca a 12 países, se creó un sitio web específico para la información de las negociaciones ${ }^{55}$. Desde distintos países se reclama que las negociaciones comerciales entre bloques se tratarían de un "libre comercio a hurtadillas" ${ }^{16}$. Se afirma que incluso la Comisión Europea, en la negociación del TTIP "... requiere de más transparencia que nunca, grupos de consumidores y activistas denuncian un sistema opaco y sesgado a favor de los grandes intereses industriales". La desconfianza de estos procesos de negociación les quita legitimidad y aleja a los ciudadanos de los procesos de integración. Algunos gremios han reclamado por la falta de transparencia en el proceso ${ }^{57}$ y se sienten "a ciegas", y las ONG que se oponen ${ }^{58}$ exigen un "TPP transparente y justo". Para eliminar esta percepción, se organizaron reuniones para transparentar, con el denominado "Cuarto Adjunto Amplio"59, recientemente lanzado con el objetivo de "propiciar la activa participación de gremios y entidades de la sociedad civil de forma permanente", durante las negociaciones del acuerdo, para entregar la información requerida para preparar estas reuniones y definir las posiciones del país. Los interesados deben expresar su interés, ya sean ONG, sindicatos, gremios, instituciones académicas u otras similares ${ }^{60}$.

Recursos ante el Consejo para la Transparencia por el Acuerdo Transpacifico y otras negociaciones:

52 En las resoluciones exentas J 1062 de 2012, J 1318 de 2012 y J 0541 de 2013. Disponible en internet: http://www.direcon.gob.cl/transparencia/PDF_Transparencia/Resol-Ex-J-0541-2013-part-ciudadana.pdf

53 Resolución Exenta n. ${ }^{\circ}$ J-0541/2013

54 Disponible en internet: www.direcon.cl

55 Disponible en internet: http://www.direcon.gob.cl/tpp/

56 Disponible en internet: http://internacional.elpais.com/internacional/2014/04/21/actualidad/1398100048_802222.html

57 Disponible en internet: http://www.elmostradormercados.cl/destacados/opinion-la-poca-transparenciade-las-negociaciones-del-tpp/

58 Disponible en internet: http://tppabierto.net/

59 Disponible en internet: http://www.direcon.gob.cl/2014/04/cerca-de-30-organizaciones-y-gremios-sonconvocados-por-la-direcon-para-lanzarproceso-de-transparencia-del-tpp/

60 Disponible en internet: http://www.direcon.gob.cl/tpp/cuarto-adjunto/ 
Daniel Álvarez Valenzuela con Direcon, Rol: C1534-12, 25/01/2013. Se requirió acceso a los documentos elaborados por un consultor en la etapa de negociación del TPP. Por tratarse de un "proceso deliberativo en curso, en el marco de las negociaciones internacionales del país, otorgándose garantía de confidencialidad a las contrapartes respecto a que la información proporcionada no será divulgada a terceros, sean estos otros Estados o la sociedad civil. En particular, en el marco del TPP existe un compromiso expreso en materia de confidencialidad definido con anterioridad al inicio de las negociaciones. Infringir dicha garantía y compromiso de confidencialidad constituiría un comportamiento de mala fe que afectaría al Estado de Chile individualmente, así como a otros países, alterándose la confianza puesta en el Estado chileno y su consecuente capacidad negociadora, produciendo con ello un detrimento en sus relaciones exteriores".

Óscar Corvalán Aracena con Direcon, Rol: C738-12, 05/09/2012. Se requirieron copias de los siguientes antecedentes: a) borradores del Tratado Multilateral denominado Trans-Pacific Partnership (TPP), del cual el Estado de Chile es parte negociante, junto a otros ocho países del Asia Pacífico, EE UU, Australia, Nueva Zelanda, Singapur y Perú, entre otros; b) estudios técnicos y económicos que justifiquen la suscripción del Trans-Pacific Partnership (TPP), y c) copia de todo otro documento que obre en poder del Ministerio de Relaciones Exteriores, que diga relación con lo solicitado, lo que fue denegado por Direcon, ya que: "ello implicaría entregar o dar acceso a los textos presentados por los gobiernos de los países participantes en esta negociación internacional, lo cual podría afectar el debido cumplimiento de las funciones de dicho servicio y el interés nacional, ya que se refieren a las relaciones internacionales del país". Como ocurre con el proceso de elaboración de cualquier tratado internacional, se desarrolla con la mayor confidencialidad, otorgando seguridad de que la información proporcionada no será divulgada a terceros, sean estos otros Estados o la sociedad civil. Es fundamental otorgar las garantías mínimas que permitan llevar a cabo el proceso dentro de un marco de confianza entre países con intereses normalmente contrapuestos. De esta forma, señala que desde el comienzo de las negociaciones se acordó entre todos los participantes del TPP que las propuestas, presentaciones y textos de negociación formulados en el marco de dicho proceso serían confidenciales. La información fue entregada por las contrapartes negociadoras, bajo el entendido de mantenerse confidencial, y hacerlo "violentaría la confianza puesta en el Gobierno chileno, dañando la capacidad negociadora de Chile y sus relaciones exteriores", importaría una conducta de mala fe ${ }^{61}$ y afectaría el objetivo de la negociación. La confidencialidad fue requerida por Nueva Zelanda y aceptada formalmente por Chile, lo que fue acreditado en el proceso; además, se trata de un proceso inconcluso de negociación y, por ello, cae en las causales establecidas en el artículo 21 n. ${ }^{\circ} 1$ letra b) de la Ley de Transparencia, cuyos requisitos copulativos establecidos por

61 Ello, además, "ha sido recogido por la Doctrina, en base a la jurisprudencia de la Corte Internacional de Justicia, en particular en la sentencia sobre los casos de la Plataforma continental del Mar del Norte, en cuanto a que el proceso de celebración de los tratados se rige por el principio de la buena fe y genera obligaciones de comportamiento de los Estados incluso durante las negociaciones". 
la jurisprudencia son: a) antecedente o deliberación previa a la adopción de una resolución, medida o política y b) que la publicidad, conocimiento o divulgación de dicha información afecte ${ }^{62}$ el debido cumplimiento de las funciones del órgano. El daño debe ser acreditado por el propio órgano que lo invoca ${ }^{63}$.

Direcon plantea que la "confidencialidad acordada respecto de las propuestas de texto de los países participantes en el TPP se apliquen incluso a las propias propuestas de Chile, que recogen en parte o se emiten en respuesta de las otras propuestas presentadas en la negociación. Así, al hacer pública su propuesta, estaría dejando entrever la posición de los otros. Sobre este punto señala que, según el requirente, se trataría de información que el Estado de Chile hace conocida a los demás países negociadores y que, por lo tanto, sería de amplio conocimiento. Tal aseveración es infundada. Chile entrega sus propuestas en el marco del TPP, con la seguridad de que estas no serán divulgadas por los otros países, y que ante una solicitud de acceso en sus respectivos países procederán a denegar la entrega de copias de las mismas. La confidencialidad en el manejo de la información que deben hacer los otros países es la contrapartida del compromiso asumido por Chile en esta materia". No respetarlo, para el Consejo: podría generar un daño específico en nuestras relaciones bilaterales, por cuanto no respetar dicho compromiso de reserva dañaría la confianza puesta en el gobierno chileno, perjudicando la capacidad negociadora de nuestro país. Del mismo modo, al encontrarse en un trámite pendiente de deliberación, "es indudable que los borradores o propuestas de texto de negociación, tanto de Chile como de los otros países participantes en la negociación del TPP, detentan el carácter de antecedente o deliberación previa de los capítulos que definitivamente formarían parte del tratado internacional que los países participantes suscribirían y que sería adoptado mediante el correspondiente decreto promulgatorio". Y que el beneficio público de conocer las propuestas de texto de negociación presentadas por Chile y los demás países participantes es menor que el daño que inevitablemente produciría su divulgación. La resolución del amparo acogió parcialmente la petición y dispuso la entrega de información pertinente y "que no afecten los bienes jurídicos amparados por las causales de secreto o reserva invocadas por la Direcon".

Daniel Álvarez Valenzuela con Ministerio de Relaciones Exteriores, Rol: C666-12, 29/08/2012, que también solicitaba copias y antecedentes de las negociaciones del TPP, con similares argumentos del amparo C738-12, ya comentado, y con la fundamentación de una filtración en el diario The New York Times, de antecedentes significativos de la negociación, fue acogido parcialmente, basado en los criterios anteriores ${ }^{64}$, y ordenó la entrega a los reclamantes del "estudio realizado por las economías APEC, sobre el impacto que tendría un TLC entre los países miembros de APEC ("Further Analytical Study in the

62 Según lo establecido en las decisiones recaídas en los amparos roles A96-09, A165-09, C193-09, C84010, C850-10, C492-11 y C929-11

63 Según lo establecido en las decisiones recaídas en los amparos Roles A1-09, A39-09, A45-09, C1233-11 y C1234-11.

64 Amparos Roles A310-09, A337-09, C382-09 y C294-11. 
Likely Economic Impact of an FTAAP — Paper", de 8 de Noviembre de 2009) y las Recomendaciones realizadas por el Consejo Empresarial de APEC - ABAC ("Building Towards the Bogor Goals with One Community - Report to APEC Economic Leaders", Singapur 2009)". Las causales de reserva del artículo 21 de la Ley de Transparencia son uno de los "conceptos jurídicos indeterminados", lo que significa, entre otras cosas, realizar un estudio "caso a caso" (FERNÁNDEZ VIVANCO) o cuyo contenido y extensión requiere ser delimitado para su aplicación al caso en concreto. Lamentablemente, nuestra Ley de Transparencia no cuenta con el grado de especificidad que hubiera sido preciso, ni tampoco estuvo la idea dentro del debate parlamentario respecto la discusión de la llamada Ley de Probidad (Ley n. $\left.{ }^{\circ} 19.653\right)^{\prime \prime}$. No basta su mera invocación como causal, sino que debe demostrarse que existe un posible daño, aplicando un test de proporcionalidad. Respecto de los antecedentes de la negociación en temas de propiedad intelectual y el comercio electrónico, "y a pesar de poder alcanzarse algunos consensos sobre ciertos asuntos, nada obsta ni inhibe a que alguno de los países participantes pueda posteriormente requerir la revisión de los textos acordados con anterioridad. Por tanto, sostiene que las propuestas sobre dichas materias, tanto de Chile como de los otros países participantes en la negociación del TPP, detentan el carácter de antecedente o deliberación previa de los capítulos que definitivamente formarían parte del tratado".

Paulo Montt Rettig y Luis Cordero Vega con Subsecretaría de Relaciones Exteriores, Direcon, Rol: C1233-11,07/03/2012. Por tratarse de una causa pendiente en el extranjero, debe distinguirse, señala el Consejo en la fundamentación del rechazo de la causal de reserva, entre documentos que dan cuenta de la estrategia jurídica, como minutas internas, informes técnicos, el expediente interno del litigio, y otros que servirán como medios de prueba. Los primeros sí son reservados, mientras que los segundos lo serían solo mientras dure la etapa probatoria. Una vez que no cumplan un rol en la defensa judicial del organismo, en el caso en comento el Ministerio de RR EE, se trata de una materia que concierne a la relación internacional del Estado y la defensa internacional de los intereses del país (dentro de la competencia de este Ministerio) y en un litigio que se sustancia en una jurisdicción extranjera, aún no concluido.

Marco Correa Pérez con Servicio Nacional de Aduanas, Rol: C1200-12, 07/12/2012. Se resolvió la solicitud de información aduanera singularizada de un ciudadano, y se mantuvo el criterio de distinguir si se trata de "naturaleza reservada" y la "expectativa de confidencialidad"65, para lo cual se siguió el precedente de los amparos A37-09 y C868-12. No basta la sola naturaleza reservada ${ }^{66}$ y que posea un valor comercial y no sea generalmente conocida ni fácilmente accesible, sino que además "el titular de la misma

65 De conformidad alcon el artículo 10 del Acuerdo relativo a la aplicación del artículo VII del Acuerdo General sobre Aranceles Aduaneros y Comercio de 1994 (GATT, de 1947) -o Acuerdo de Valoración Aduanera o de Valor de la OMC. 
adopte todas las medidas, necesarias y eficaces, para mantener en secreto o reserva dicha información"67.

Negociaciones de la Alianza del Pacífico ${ }^{68}$. De especial interés han resultado los avances de la Alianza, que reúne a Chile, Colombia, Perú y México, que cuenta con un importante número de 30 observadores. En el Protocolo Adicional al Acuerdo Marco Alianza del Pacífico ${ }^{69}$, se indican sus ventajas desde la transparencia: contratación pública, que mejora los estándares para todos los miembros, en trato nacional, no discriminación y transparencia en ámbitos que no todos tenían; obstáculos técnicos al comercio (TBT): establece mejores disciplinas de transparencia y procedimientos de conformidad y cooperación regulatoria, creando un comité de monitoreo e implementación; medidas sanitarias y fitosanitarias (SPS): permite mejorar los actuales canales de información y bases científicas, con una mejor cooperación entre agencias estatales y con un comité técnico para el seguimiento e implementación.

\section{COMISIÓN NACIONAL DE DISTORSIONES DE PRECIOS (CNDP)}

Se trata de la entidad nacional que resuelve, en un cuasi juicio de carácter administrativo, la recomendación de aplicación de medidas, tales como derechos compensatorios o antidumping o salvaguardias. Para las dos primeras, existen maneras más o menos objetivas para la determinación de la práctica desleal, y en la que debe probarse el daño; para la tercera (salvaguardias), se trata de una cláusula de escape cuando existe una situación abrupta e inesperada de un aumento de las importaciones, en una proporción que puede producir un daño irreparable. En todos los casos, la determinación se lleva ante una revisión externa judicial. Se trata de procedimientos que deben ventilar información relevante de las empresas, como su estructura de costes para determinar el dumping, o la devolución de valores que han sido rebajados de impuestos ilegítimamente. En cada caso, se debe evaluar la conveniencia de mantener la reserva de los análisis e información que pudiera perjudicar a la empresa o industria que se somete a la evaluación. Sin embargo, los niveles de transparencia de estos procesos, que habitualmente no cuentan con representación legal, quedan muchas veces sometidos a decisiones que ponderan la influencia y presión de las industrias que solicitan la protección, y un sobreprecio que termina gravando a los consumidores nacionales. En los debates ante las entidades nacionales (local authority para la OMC) que determinan la aplicación de una medida trade remedies, como salvaguardias, antidumping o derecho compensatorio, se realiza un análisis que comúnmente no permite conocer los elementos esenciales para una adecuada

67 Alberto Rodríguez Bosshard con Servicio Nacional de Aduanas, Rol: C781-11, 28/10/2011.

68 Disponible en internet: http://www.direcon.gob.cl/tpp/

69 Disponible en internet: http://www.direcon.gob.cl/wp-content/uploads/2014/02/Protocolo-de-la-Alianzadel-Pac\%C3\%ADfico.pdf 
transparencia, como en el caso de la Comisión Nacional de Distorsiones de Chile ${ }^{70}$. Existe un proceso que no permite conocer buena parte de lo que fundamenta una medida que puede perjudicar a los consumidores, aunque el argumento que se emplee sea la protección de la industria nacional. La existencia de lobbies exige la mayor transparencia posible y que el procedimiento ante la CNDP procure dar garantías de transparencia. Cuando se realizan determinaciones de afección de alguna industria, establece lo siguiente: "A objeto de garantizar la máxima transparencia del proceso de una investigación, así como para facilitar la participación de todas las partes interesadas, la CNDP da público conocimiento de todas las determinaciones preliminares o definitivas, positivas o negativas y del término de una investigación a través de las publicaciones correspondientes en el Diario Oficial y de las actas de las sesiones de la Comisión que, una vez aprobadas, son públicas y que se mantienen para consulta en la Biblioteca del Banco Central. No obstante lo anterior, la Comisión mantiene en forma confidencial aquella información que haya sido considerada como tal en su momento". La reserva de los antecedentes presentados ante la CNDP requiere ser solicitada expresamente por la parte que los somete, y las causas justificadas que expliquen su reserva ${ }^{71}$, en caso contrario, deben acompañarse de una versión pública. Las actas de sus sesiones se encuentran disponibles, y aquellas en las que se resuelven materias relevantes se publican en el Diario Oficial, sin embargo, los fundamentos de las resoluciones no son extensivos, y los antecedentes técnicos quedan, muchas veces, sin poder conocerse. El Grupo Especial ya estimó que no bastaba "poner a disposición del público", y que también se publicarán las sesiones, aunque no se determinó su contenido ${ }^{72}$.

La decisión de iniciar una investigación se debe informar al Ministerio de Relaciones Exteriores, para la notificación a los países potencialmente afectados. La apertura de una investigación se publicará en el Diario Oficial, con la información pertinente especificada ${ }^{73}$; asimismo, los integrantes del CNDP decidieron, en marzo de $2004^{[74]}$, publicar en el sitio web institucional, las minutas relacionadas con las investigaciones.

Información Confidencial y su tratamiento. Con respecto a la información confidencial, 30 días después de su publicación, los interesados facilitarán a la Secretaría Técnica toda la información pertinente, con la especificación de ser tratada de forma confidencial. Dentro de los 90 días siguientes a la publicación, se debe resolver, en todos los casos, con una publicación en el Diario Oficial con una de las siguientes alternativas: i) si se estableció una distorsión, entonces el CNDP recomendará al Presidente la aplicación de una medida y su proporción, ii) cuando la evidencia no es considerada del todo suficiente para demostrar la existencia de distorsiones de precios o el daño de la misma, deberá decidir y enviar una notificación a la otra parte ${ }_{i}$ iii) el CNDP, en cualquier momento antes 
de la determinación final, podrá solicitar del Presidente de la aplicación de una medida provisional dentro de los 60 días.

La divulgación de cierta información podría, eventualmente, beneficiar a los competidores o generar un daño significativo a las partes ${ }^{75}$; por lo tanto, la preocupación especial y adecuada protección de cierta información (que por su naturaleza se considere confidencial o que aporten las partes sobre una base confidencial) deben ser tratadas adecuadamente. Dicha información no puede ser divulgada sin el consentimiento expreso de la parte que la presente ${ }^{76}$. Toda esa información debe ir acompañada de un resumen o una justificación de la confidencialidad. Esto provoca un problema: que dicha información confidencial se hace no revisable y no verificable. En cuanto a eso, el Grupo Especial consideró que la disposición del artículo 3.2 del Acuerdo sobre Salvaguardias faculta a las autoridades nacionales para una amplia discreción en lo que respecta a la determinación de la divulgación de información que se considera confidencial, [pero, la] no divulgación de información crucial para la determinación del daño puede comprometer la transparencia de la investigación, provocando quejas y disputas ${ }^{77}$.

Audiencia Pública. Los interesados deberán disponer de tiempo y oportunidad para la defensa y la réplica de los argumentos de interés adverso. Las autoridades, siempre que se considere práctico, proporcionarán la oportunidad de revisar toda la información pertinente que no sea confidencial ${ }^{78}$. El Presidente del CNDP determinará la duración, la estructura de la audiencia y el tiempo disponible para las partes. Esta información se facilitará con prontitud a todas las partes interesadas. Antes de la aplicación de la medida definitiva (medida de salvaguardia, antidumping o compensatorio investigación deberes), el CNDP informará la fecha y el lugar de la audiencia pública a todas las partes interesadas en presentar pruebas y expresar sus planteamientos y respuestas respecto a la información de otras partes. Cada parte deberá presentar por separado; para tal fin, se concedió a las partes el mismo tiempo, de acuerdo con su posición a favor o en contra de la medida. El orden de aparición en la audiencia será el mismo que el establecido en la minuta anterior. Las partes tendrán el derecho de réplica, con el único fin de aclarar las cuestiones relacionadas con la información y las pruebas presentadas por los participantes anteriores. Las minutas proporcionan información limitada, como la lista de participantes y una breve presentación escrita ${ }^{79}$. Los representantes de los gobiernos extranjeros que deseen participar deberán tener un tiempo similar para las partes involucradas, para que conste por cuenta de terceros que no estuvieron presentes en la audiencia pública.

77 LEE, YONG-SHIK. Safeguard Measures: Why are they not Applied Consistently with the Rules?, Journal of World Trade 36(4) 2002, p. 659 (Wheat Gluten, WT/DS166/R para 8.11).

78 Acuerdo Antidumping, artículo 6.2 y SCM Agreement, artículo 12.3.

79 La estructura más común de las minutas es la siguiente: Antecedentes I. General; II. Análisis de las importaciones; III. Daño, y relación causal, incluyendo los precios internos, empleo, productividad, capacidad de producción máxima, ventas domésticas, producción total, consumo, supuesta participación de las importaciones "en el presunto consumo y los precios internacionales". 
Las partes pueden disponer, ya sea antes o el mismo día de la audiencia, de una presentación por escrito que contenga un resumen de la presentación de la que se habla en la audiencia. Las partes proporcionarán información sobre sus actividades profesionales, así como su interés en el caso.

\section{TRANSPARENCIA VS DEBIDA RESERVA}

Fiscalía Nacional Económica ${ }^{80}$. El órgano que debe velar por las normas de competencia y evitar distorsiones de mercado es la Fiscalía Nacional Económica (FNE), la que debe desarrollar investigaciones, recibir denuncias, solicitar estudios muy complejos, además de lidiar con información económica y comercial muy sensible para las empresas. En el transcurso de sus investigaciones y durante el proceso de acusación, se ha visto expuesta a una interesante cuestión respecto de la transparencia, que descansa en la garantía constitucional y en la normativa de la función pública. Pero puede verse expuesto el propio desempeño, el éxito de su función y, peor aún, dañar al denunciante. Respecto de la transparencia activa, esto es, la información que se muestra en el portal web de la institución ${ }^{81}$, se ha desarrollado una labor proactiva y que facilita el conocimiento del rol y la actividad que está desarrollando la FNE, así como informes y minutas que fundamentan la resolución de no continuar algún proceso. Asimismo, se ha incluido información que sirve para ilustrar las pautas y criterios empleados por la Fiscalía a través del "Instructivo interno para el desarrollo de investigaciones de la FNE". En cada caso, se ha velado por la protección de cualquier información que, por su condición, pudiere ser sensible o relevante para alguna industria, evitando causar un perjuicio, pero manteniendo una política acorde con un acceso abierto y ampliado de una temática que, por su naturaleza, despierta muchas desconfianzas y suspicacias. La naturaleza de la información implica un especial cuidado y protección de la reserva de las denuncias y los antecedentes comerciales relevantes. Ello, en principio, puede ser definido por el propio Fiscal, y de oficio o a petición de un interesado, algunas partes del expediente de investigación pueden mantener confidencialidad. Es entendible que una prudente garantía de protección de la información asegura al denunciante, sean empresas o personas naturales. Como ha sostenido el Consejo ${ }^{82}$, debilitaría su facultad fiscalizadora ${ }^{83}$.

80 Irarrázabal, Felipe y Avendaño, Andrea. "La mirada de la Fiscalía Nacional Económica sobre el equilibrio entre la transparencia de la función investigadora y litigante de un órgano persecutor".

81 Disponible en internet: http://www.fne.cl/

82 C567-09, C518.

83 "...la divulgación de la investigación sin que aún se haya adoptado decisión sobre el particular, produciría un perjuicio o afectación en el debido cumplimiento de las labores encomendadas a la FNE, toda vez que dejaría en evidencia las diligencias decretadas por ésta, lo que, a su vez, permitiría conocer su línea investigativa y sus eventuales objetivos y resultados, como también las consideraciones que ha tenido a la vista, pudiendo presumirse, así, la eventual medida a adoptar, lo que debilitaría manifiestamente su facultad fiscalizadora." (C567-09; C518-09). 
El Tribunal de la Libre Competencia puede, al conocer los asuntos que se le sometan, decretar la reserva de la información (Decreto Ley 211) y también mantener la reserva cuando se le presente en este carácter (Auto Acordado N15/2012 del TDLC). La dificultad, expresa Felipe Irarrázabal, está primero en "conciliar adecuadamente el principio de publicidad de la información en poder de la FNE y el derecho de acceso, sin lesionar o menoscabar la propia labor investigativa", evitando alertar a los investigados y, en definitiva, debilitar el caso, ya que existe una disparidad de las partes. Al ser la Fiscalía el ente investigador y persecutor, se encuentra expuesto a solicitudes de transparencia pasiva, y ante la desventaja procesal de revelar a la contraparte aspectos críticos para el desenvolvimiento del caso en una etapa que bien podría afectar el interés público que defiende la FNE. Los casos en que se ha rechazado el acceso solicitado se refieren a materias de diversa índole ${ }^{84}$ : antecedente necesario para una defensa judicial ${ }_{;}$antecedentes o deliberaciones previas a la adopción de una resolución, medida o política, denuncias y su contenido o identidad del denunciante y la información entregada por empresas o particulares; protección de derechos de carácter comercial o económico. Todas han sido, hasta la fecha, acogidas como causales justificadas de reservas por diversos recursos de amparo ante el Consejo para la Transparencia. Uno de los pocos casos hasta ahora rechazado en que se ha invocado causal de reserva fue uno en que la FNE, encontrándose en diligencias judiciales pendientes, se excusó de exhibir informes en derecho encargados por la FNE ${ }^{85}$ y títulos o temas de informes en derecho encargados en un período de tiempo determinado. Para el Fiscal Nacional, ello constituía "una afrenta a su estrategia de defensa judicial", que implica una "asimetría de las partes", ya que una de ellas podría acceder, aun de modo genérico, a antecedentes de defensa de la contraria, por tratarse de una entidad obligada a dar acceso irrestricto a la transparencia. Por otro lado, se trata de revelar información comercial que no sería significativa y no afectaría a las personas o entidades que la aportaron; sin embargo, expone el Fiscal, tener acceso a esa información podría generar desconfianza en posibles aportantes futuros de información y afectar el cumplimiento de la función de la FNE.

Semillas transgénicas y transparencia. Las normas de rotulación de alimentos comúnmente adolecen de un bajo nivel de descripción. Luego de sendos rechazos en EE $\mathrm{Uu}^{86}$ por la propuesta de etiquetar los alimentos que contienen componentes de origen transgénico, facilitando a los consumidores tomar una decisión, en coincidencia con lo que la Comisión Europea denomina la coexistencia ${ }^{87}$, como la capacidad de escoger entre cultivos convencionales, ecológicos y modificados genéticamente, cumpliendo las normas de etiquetado y de pureza (2003/556/CE), sin transparencia y trazabilidad no puede existir un ejercicio legítimo de la libertad de elegir. De este modo, se acogió 
el recurso ${ }^{88}$ que solicitó conocer el lugar donde se encontraban los cultivos de organismos genéticamente modificados, en los que se debatió respecto del carácter público de dicha información y la posibilidad de afectación de un derecho de propiedad o a la vida o reserva de privados, lo que fue desechado como causal aplicable de reserva en este caso, y se ordenó "informar la ubicación exacta y el nombre del propietario y/o entidad responsable autorizados para cultivar y acopiar semilla transgénica de exportación como también para la experimentación científica en vegetales transgénicos".

\section{CONCLUSIONES}

La sociedad y la tecnología han permitido el desarrollo de nuevos canales de participación y de organización. Las ONG, que hasta hace poco eran las únicas que tenían la capacidad de encauzar las sensibilidades ciudadanas frente a las negociaciones de los TLC, hoy se han visto aumentadas y superadas por las redes sociales. Los ciudadanos son también consumidores y tienen expectativas de ser tomados en cuenta, ya no solo de ser informados de los avances de las negociaciones y de la implementación de los acuerdos comerciales. Hoy, exigen tener un rol en la definición de las políticas públicas, quieren ser oídos y que sus opiniones, más o menos fundadas técnicamente, sean consideradas al aprobar las políticas públicas. Las experiencias recientes en el debate energético son una muestra clara de que las redes sociales no son una fuente de pasividad y conformidad ciudadana. Muy por el contrario, los ciudadanos y consumidores demandan y esperan obtener adecuados mecanismos de participación en una democracia directa, una democracia líquida y en la política de la integración comercial. Demandan una nueva manera de participación que denominaremos "integración líquida". Ya no es suficiente la transparencia activa que otorga legitimidad democrática y permite un mayor accountability. Tampoco parece ya ser suficiente que existan canales de transparencia pasiva, en los que hay interacción al requerir una información particular, la que permite dar mejores estándares de participación e involucramiento, que también implican una mejor gobernanza y control ciudadano de la actuación de la administración nacional y los organismos multilaterales. Hoy, los ciudadanos, algunos más organizados que otros, exigen ser incluidos en el proceso de toma de decisiones, de manera directa y en tiempo real (y porque la tecnología así lo permite), de las negociaciones y acuerdos, y ello implica una nueva manera de ejercer los derechos democráticos, de escrutinio público y de gobernanza. Lo que no sabemos bien es cómo los organismos multilaterales, los gobiernos y las empresas asumirán estos complejos desafíos, restringiendo al mínimo las causales de reserva o denegación de acceso. El rol fundamental de las entidades que tutelan el ejercicio de la transparencia a nivel nacional (CPLT) y a nivel internacional (tribunales internacionales) nos plantea la necesidad de velar por que las delimitaciones 
de este derecho sean objetivas, previsibles y restringidas, de modo que concilien lo que está detrás de la transparencia: el ejercicio de derechos fundamentales.

BIBLIOGRAFÍA

Ahlborn, Christiane y Headen Pfitzer, James. Transparency and Public Participation in wto Dispute Settlement. Center for International Environmental Law, 2009.

Ala'I, PADIDEH. From the Periphery to the Center? The Evolving WTO Jurisprudence on Transparency and Good Governance. J Int. Economic Law (2008) 11 (4): 779-802.

Bellei Tagle, Carlos. Amicus Curiae Briefs Before wTo Dispute Settlement System: Welcome Friends? Godina 2012/13, broj 2, Year 2012/13, no. 2.

BELLO, WaLDEN. Lack of Transparency in the WTO. Development Dialogue 2002:1.

Bernstein, Steven y Hannah, Erin. Non-State Global Standard Setting and the WTO: Legitimacy and the Need for Regulatory Space. J Int. Economic Law (2008) 11 (3): 575608.

BONZON, YVES. Institutionalizing Public Participation in WTO Decision Making: Some Conceptual Hurdles and Avenues. J Int. Economic Law (2008) 11 (4): $751-777$.

Changing relationships between International Non-Governmental Organizations and the United Nations (http://www.uia.org/doc-archive).

Charnovitz, Steve. The WTO and Cosmopolitics. J Int. Economic Law (2004) 7 (3): 675-682.

Charnovitz, Steve. The WTo's Environmental Progress. J Int. Economic Law (2007) 10 (3): 685-706.

Charnovitz, Steve. Transparency and Participation in the World Trade Organization. 56 Rutgers L. Rev. 927, 2004.

Cheyre, Juan Emilio y Cobo, Nicolás (eds.). Transparencia en Chile, logros y desafíos a cinco años de vigencia de la Ley. Orjikh Editores Ltda, 2014.

DOWNES, CHRIS. The Impact of WTO Transparency Rules: Is the 10,000th SPS Notification a Cause for Celebration? A Case Study of Eu Practice. J Int. Economic Law (2012) 15 (2): 503-524.

Ecologic - Institute for International and European Environmental Policy. Participation of NonGovernmental Organisations in International Environmental Governance: Legal Basis and Practical Experience.

Ehring, Lothar. Public Access to Dispute Settlement Hearings in the World Trade Organization. Journal of International Economic Law 11(4), 1021-1034, 2008.

FLIESS, B. Transparency of Export Restrictions: A Checklist Promoting Good Practice. OECD Trade Policy Papers, no. 164. OECD Publishing, 2014.

GaO, HENRY y LiM, C. L. Saving the WTO from the Risk of Irrelevance: The WTO Dispute Settlement Mechanism as a 'Common Good' for RTA Disputes. J Int. Economic Law (2008) 11 (4): 899-925.

García MaCho, Ricardo (ed.9. Derecho Administrativo de la información y administración transparente. Marcial Pons, 2010.

Guichot, EmiLio. Transparencia y acceso a la información en el derecho europeo. Cuadernos Universitarios de Derecho Administrativo. Editorial Derecho Global, 2011.

Halle, MARK y Wolfe, Robert. A new approach to transparency and accountability in the WTO. Issue Brief 06 Entwined, 2010. 
Hoekman, Bernard. Operationalizing the Concept of Policy Space in the WTO: Beyond Special and Differential Treatment. J Int. Economic Law (June 2005) 8 (2): 405-424.

JACKSON, J.H. The WTO 'constitution' and proposed reforms: seven 'mantras' revisited. J Int Economic Law (2001) 4 (1): 67-78.

LejÁRRAGA, I. Multilateralising Regionalism: Strengthening Transparency Disciplines in Trade, OECD Trade Policy Papers, n. ${ }^{\circ}$ 152. OECD Publishing, 2013.

LejÁRraga, I. y ShePHerd, B. Quantitative Evidence on Transparency in Regional Trade Agreements, OECD Trade Policy Papers, n. ${ }^{\circ}$ 153. OECD Publishing, 2013.

Marceau, Gabrielle y Hurley, Mikella. Transparency and Public Participation in the wTO: A Report Card on WTO Transparency Mechanisms 4(1) Trade L. \& Dev. 19 (2012).

Petersmann, ERnSt-Ulerich. Challenges to the Legitimacy and Efficiency of the World Trading System: Democratic Governance and Competition Culture in The Wto: Introduction and Summary. J Int. Economic Law (2004) 7 (3): 585-603.

Petersmann, ERnSt-Ulrich. wTo Negotiators Meet Academics: The Negotiations on Improvements of the WTO Dispute Settlement System. J Int. Economic Law (2003) 6 (1): 237-250.

Ruddy, Brendan. The Critical Success of the wTO: Trade Policies of the Current Economic Crisis. J Int. Economic Law (2010) 13 (2): 475-495.

SANDFORD, IAIN. The Importance of Transparency in wTO Dispute Settlement. Lowy Institute Conference on Enhancing Transparency in the Multilateral Trading System, 2007.

Steger, Debra P. Introduction to the Mini-Symposium on Transparency in the WTO. J Int. Economic Law (2008) 11 (4): 705-715.

Steger, Debra P. The Future of the wTO: The Case for Institutional Reform $i$ J Int. Economic Law (2009) 12 (4): 803-833.

Umbricht, Georg C. An 'Amicus Curiae Brief' on Amicus Curiae Briefs at the WTo. J Int. Economic Law (2001) 4 (4): 773-794.

Van Den Bossche, Peter. NGo Involvement in the WTO: A Comparative Perspective. Journal of International Economic Law 11(4), 717-749, 2008.

Villegas Delgado, CÉSAR. La preeminencia del Derecho en el Derecho Internacional. Thomson Reuters Aranzadi, 2013.

Wolfe, Robert. Decision-Making and Transparency in the 'Medieval' wTO: Does the Sutherland Report have the Right Prescription? European Journal of International Relations (2010) 16 (4): 563-587.

WOLFE, ROBERT. Regulatory transparency, developing countries and the WTO. World Trade Review, 2003, 2: 2, 157-182.

WTO Discussions Narrow in on Transparency and Civil Society Relations. Bridges, vol. 2, n. ${ }^{\circ}$ 5, 1998. 